\title{
Artesunate attenuates foam cell formation by enhancing cholesterol efflux
}

\author{
Yan Qian ${ }^{1}$, Li Xia ${ }^{2}$, Lai Wei ${ }^{1}$, Weiwei Jiang ${ }^{1}$ \\ ${ }^{1}$ Department of Pharmacy, The Second Affiliated Hospital, Chongqing Medical University, Chongqing, China; ${ }^{2}$ Department of Liver Disease, \\ Chongqing Traditional Chinese Medicine Hospital, Chongqing, China \\ Contributions: (I) Conception and design: W Jiang; (II) Administrative support: L Xia; (III) Provision of study materials or patients: W Jiang; (IV) \\ Collection and assembly of data: Y Qian, L Wei; (V) Data analysis and interpretation: Y Qian; (VI) Manuscript writing: All authors; (VII) Final \\ approval of manuscript: All authors. \\ Correspondence to: Weiwei Jiang. Department of Pharmacy, The Second Affiliated Hospital, Chongqing Medical University, 76 Linjiang Road, \\ Yuzhong District, Chongqing 400010, China. Email: 304035@cqmu.edu.cn.
}

Background: Atherosclerosis is the main cause of many cardiovascular diseases and the second leading cause of death in elderly people. The formation of intimal macrophage-derived foam cells is a major feature of early atherosclerotic lesions. Little is known about the effects of artesunate (ART) on macrophage-derived foam cell formation.

Methods: Oil red O staining was employed to detect foam cell formation; colorimetric analysis was employed for cholesterol measurement; quantitative real time polymerase chain reaction (qRT-PCR) and western blot analysis were employed to assess messenger RNA (mRNA) and protein expression, respectively; enzyme-linked immunosorbent assay (ELISA) analyses were used to observe interleukin 6 (IL-6) and tumor necrosis factor- $\alpha$ (TNF- $\alpha$ ) release; and 3-(4,5-dimethylthiazol-2-yl)-2,5-diphenyl tetrazolium bromide (MTT) assays were used to examine cell viability.

Results: It was revealed that ART attenuated oxidized low-density lipoprotein (ox-LDL)-induced foam cell formation from THP-1-derived macrophages by decreasing cholesterol accumulation, and the effect might have occurred via enhanced cholesterol efflux. Additionally, ART decreased toll-like receptor 4 (TLR4) expression, increased adenosine triphosphate (ATP)-binding cassette transporter A1 (ABCA1) and ATPbinding cassette transporter G1 (ABCG1) expression, and reduced the secretion of IL- 6 and TNF- $\alpha$.

Conclusions: This study showed that ART attenuated the ox-LDL-induced formation of foam cells from THP-1-derived macrophages by increasing ABCA1 and ABCG1 expression via inhibiting TLR4 expression and reducing TNF- $\alpha$ and IL-6 secretion from macrophages induced by ox-LDL, which ultimately decreased the accumulation of cholesterol. It is worthwhile further investigate ART as a potential drug for the treatment of atherosclerosis.

Keywords: Artesunate (ART); atherosclerosis; foam cell; cholesterol efflux

Submitted Jun 07, 2021. Accepted for publication Aug 11, 2021.

doi: 10.21037/atm-21-3551

View this article at: https://dx.doi.org/10.21037/atm-21-3551

\section{Introduction}

Atherosclerotic diseases are important causes of death and disability in patients, which develop throughout life through a multi-step process $(1,2)$. The formation of intimal macrophage-derived foam cells is a major feature of early atherosclerotic lesions (3). Excessive intake of oxidized low-density lipoprotein (ox-LDL), excessive cholesterol esterification, and insufficient cholesterol release cause the accumulation and storage of cholesterol esters. Cholesterol esters are stored as cytoplasmic lipid droplets and ultimately lead to foam cell formation. Therefore, balancing 
cholesterol influx, efflux, and esterification in macrophages is critical for avoiding lipid overload and the formation of atherosclerotic plaques $(3,4)$.

Very low-density lipoprotein receptor (VLDL-R), scavenger receptors (SRs), class A SR (SR-A), and CD36 are critical for internalizing of modified LDL (such as ox-LDL) in macrophages, which increases cellular accumulation of cholesterol. In contrast, reverse cholesterol transporters (RCTs), such as adenosine triphosphate (ATP)-binding cassette transporter G1 ABCG1, ATP-binding cassette transporter A1 ABCA1, and clearance receptor BI (SR$\mathrm{BI})$ are responsible for cholesterol efflux in macrophages. Cholesterol esterification is mainly controlled by acyl coenzyme A:cholesterolacyltransferase-1 (ACAT1) and neutral cholesterol ester hydrolase (nCEH) $(3,4)$. When cholesterol influx or esterification increases or cholesterol efflux decreases, macrophages become lipid-filled foam cells.

Atherosclerosis is also a chronic inflammatory process. Macrophage-derived foam cells produce various chemokines and cytokines during lipid accumulation, inducing inflammation (5). Toll-like receptors (TLRs) play important roles in the pathological process of inflammatory diseases, such as coronary artery disease (CAD). The TLRs are expressed in many immune cells, and each receptor initiates a corresponding inflammatory signal after detecting the appropriate pathogen-associated molecular pattern (PAMP) (6). In addition, in the absence of exogenous infections, the TLR signaling cascade can also be activated by a variety of host-derived molecules. Among the TLRs, TLR4 is the chief mediator of foam cell formation (7).

Artemisinin is a commonly used antimalarial drug that was first extracted from the medicinal plant Artemisia annua L. in 1971. Artemisinin is a novel sesquiterpene lactone with a peroxy bridge structure (8). Artesunate (ART) is a watersoluble cysteine derivative of artemisinin. In addition to its antimalarial effects, ART has been demonstrated to have anticancer, antiangiogenic, and anti-inflammatory effects (9-13). In our previous studies, we found that ART could decrease the progression of atherosclerotic lesion formation by inhibiting TLR4 expression and proinflammatory cytokine release (14). However, little is known about the role of ART in foam cell formation and whether the effect of ART on TLR4 expression influences the formation of foam cells. Therefore, in this study, we examined the role of ART in foam cell formation.

We present the following article in accordance with the MDAR reporting checklist (available at https://dx.doi. org/10.21037/atm-21-3551).

\section{Methods}

\section{Materials}

\section{Reagents}

In this study, we used injectable ART (injection preparation, Guilin Pharma Corp, Guangxi, People's Republic of China National Medicine Standard H10930195) with no endotoxin detected. We obtained 3-(4,5-dimethylthiazol2-yl)-2,5-diphenyl tetrazolium bromide (MTT) and lipopolysaccharide (LPS) from Sigma-Aldrich (St. Louis, MO, USA); ox-LDL from Yiyuan (Guangzhou, Guangdong, China); and Oil Red O from Yeasen (Catalog No YB-002-1, Shanghai, China). Enhanced chemiluminescence (ECL) reagents were provided by Pierce, Inc. (Rockford, IL, USA). A cholesterol assay kit was provided by Applygen (Beijing, China). Human enzyme-linked immunosorbent assay (ELISA) kits for interleukin 6 (IL-6) and tumor necrosis factor- $\alpha(\mathrm{TNF}-\alpha)$ were provided by Boster Ltd. (Wuhan, Hubei, China). Avian myeloblastosis virus (AMV) reverse transcriptase and T4 polynucleotide kinase were provided by Promega (Madison, WI, USA). Quantitative real-time polymerase chain reaction (qRT-PCR) master mix was provided by ToYoBo Ltd. (Osaka, Japan). All primers were synthesized by Invitrogen Ltd. (Shanghai, China). Cell culture medium and fetal bovine plasma were provided by Invitrogen (Carlsbad, CA, USA). The total protein extraction kit was provided by BestBio Ltd. (Shanghai, China). Antibodies for western blotting were provided by Santa Cruz Biotechnology Inc. (Santa Cruz, CA, USA).

\section{Cells}

Human monocyte THP-1 (CLS Cat\# 300356) cells were provided by the American Type Culture Collection (Manassas, VA, USA). The THP-1 monocyte culture was performed in Roswell Park Memorial Institute (RPMI)1640 (Hyclone, Logan, UT, USA) with 10\% fetal bovine serum (FBS) (Hyclone). Depending on the experiment, THP-1 cells were seeded at a density of $5.0 \times 10^{5}$ cells $/ \mathrm{mL}$ in 96-, 24-, or 6-well plates. Pretreatment with $80 \mathrm{nM}$ PMA (Sigma-Aldrich, St. Louis, MO, USA) for $72 \mathrm{~h}$, to differentiated the THP-1 monocytic cells into adherent macrophages. After that, replaced the medium containing phorbol 12-myristate 13-acetate (PMA) with RPMI-1640 containing $10 \%(\mathrm{v} / \mathrm{v})$ FBS. Before treatment, adherent 
Table 1 Primer sequences used for qRT-PCR

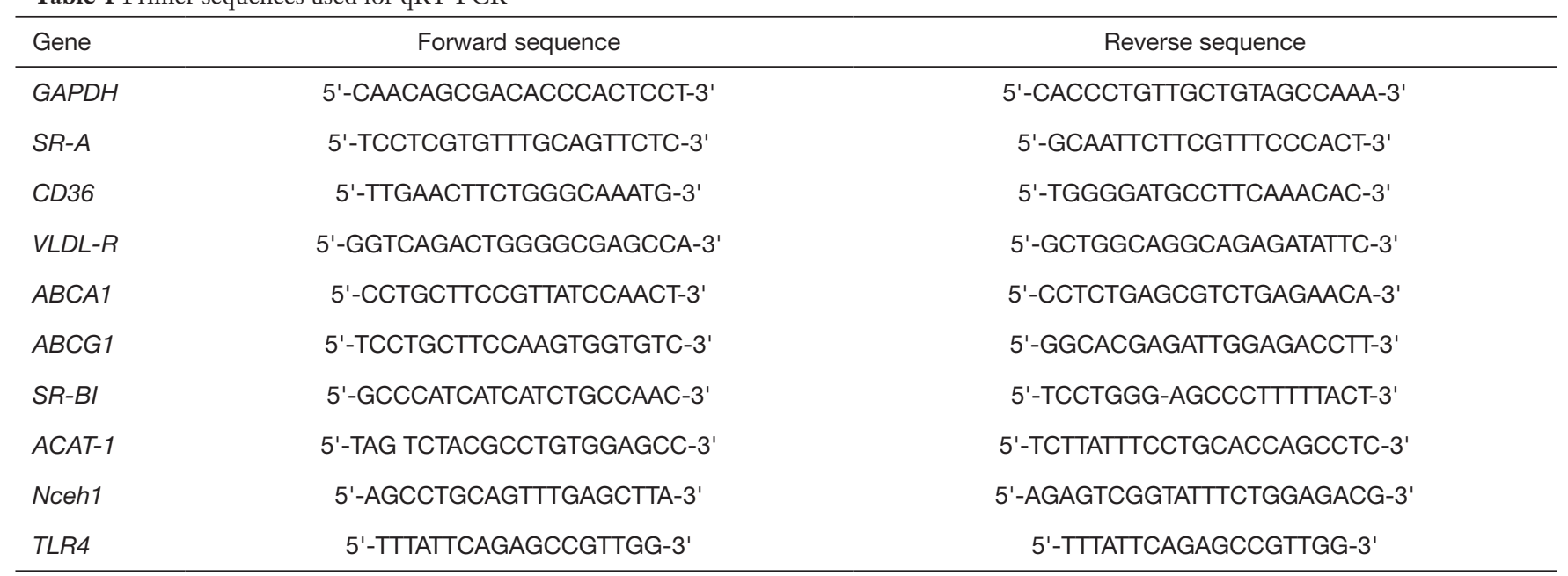

qRT-PCR, quantitative real-time polymerase chain reaction.

macrophages were cultured for an additional $24 \mathrm{~h}$.

\section{Study methods}

\section{Cell treatments}

The cells were diluted in phosphate-buffered saline (PBS) with $0.4 \%$ trypan blue and counted with a hemocytometer.

The THP-1-derived macrophages $\left(5.0 \times 10^{5}\right.$ cells $/ \mathrm{mL}$, $2 \mathrm{~mL}$ ) were incubated in 6-well plates until adherent. Then, the cells were washed twice, and different concentrations of $\operatorname{ART}(0,1.5,5$, and $15 \mu \mathrm{g} / \mathrm{mL})$ were added. After $12 \mathrm{~h}$ of incubation, the cells were treated by ox-LDL $(100 \mu \mathrm{g} / \mathrm{mL})$ for another $24 \mathrm{~h}$. After treatment, we collected the supernatants for cytokine assays, oil red $\mathrm{O}$ staining, and cholesterol measurement.

\section{Oil red O staining}

Oil red O staining was employed to detect lipid accumulation in foam cells. Briefly, the cells were first fixed in $4 \%$ frozen formaldehyde for $30 \mathrm{~min}$ and then washed with PBS. Then, the cells were incubated in propylene glycol for $5 \mathrm{~min}$ and $0.5 \%(\mathrm{w} / \mathrm{v})$ oil red $\mathrm{O}$ solution for $15 \mathrm{~min}$. Excess water was evaporated at $60{ }^{\circ} \mathrm{C}$. Next, the cells were washed with $85 \%$ propylene glycol for $5 \mathrm{~min}$ and then washed with PBS 5 times. After washing, the foam cells were photographed with a microscope.

After the extraction was completed, the density of the total neutral lipid content of the cells was measured using a microplate reader. The absorbances of the standard and samples were measured against the blank at $540 \mathrm{~nm}$.

\section{Cholesterol colorimetric assay}

The cholesterol content in macrophages was quantitatively measured by enzymatic colorimetric assays according to the manufacturer's instructions.

First, the cells were washed with PBS, then incubated in lysis buffer for $10 \mathrm{~min}$. Then, $10 \mu \mathrm{L}$ was added of each sample (cell lysate), standard (cholesterol $5 \mathrm{mmol} / \mathrm{L}$ ), and blank (distilled water) to the prelabeled tubes, and $190 \mu \mathrm{L}$ of working reagent was added. These reactions were mixed thoroughly and incubated at $37^{\circ} \mathrm{C}$ for $20 \mathrm{~min}$. Finally, the absorbances of the standard and samples were measured against the blank in a fluorescence microplate reader at $550 \mathrm{~nm}$. The total cholesterol of the samples was calculated based on the corresponding absorbances and the standard.

\section{Messenger RNA (mRNA) expression analysis}

As previously reported, total RNA was isolated, and the mRNA levels of specific genes were analyzed using qRTPCR (14). Total RNA was extracted from $1.0 \times 10^{6}$ cells and then reverse-transcribed to circular DNA (cDNA). The qRT-PCR was performed using SYBR Green and an ABI PRISM 7500 Sequence Detection System (Applied Biosystems, Waltham, MA, USA). The cDNA was amplified by PCR with the respective primers (Table 1).

\section{Protein expression analysis}

Western blot analysis of TLR4, ABCA1, ABCG1, and SR-BI was performed using conventional methods (15). Sodium dodecyl sulfate polyacrylamide gel electrophoresis (SDS-PAGE) was used to separate the same amount of 
protein from whole cell lysates, and then transferred to nitrocellulose membrane (Bio-Rad, Hercules, CA, USA). After blocking with $5 \%(\mathrm{w} / \mathrm{v})$ skim milk, the blots were incubated with the primary antibodies and then secondary antibodies. The blots were detected with ECL according to the manufacturer's instructions.

\section{Cytokine analysis}

In accordance with the manufacturer's guidelines, ELISA kits were used to analyze the culture supernatant for IL-6 and TNF- $\alpha$, respectively.

\section{Cytotoxicity assay}

Cytotoxicity was measured by MTT assay. Then, the absorbance was measured at $490 \mathrm{~nm}$ using a microplate reader.

\section{Statistical analysis}

Statistical analyses were performed using the software SPSS 10.0 (SPSS Inc., Chicago, IL, USA). All data were presented as the means \pm standard deviation (SD) of 3 independent experiments. The differences among groups were analyzed by Student's $t$-test. Values of $\mathrm{P}<0.05$ were considered statistically significant.

\section{Results}

\section{ART attenuated the ox-LDL-induced formation of foam cells in THP-1-derived macrophages}

The accumulation of lipids in macrophages is important for the development of macrophage-derived foam cells. To clarify the effects of ART on lipid accumulation and foam cell formation, THP-1-derived macrophages were mixed with ox-LDL with or without ART. Lipid droplet staining was significantly increased by ox-LDL loading, indicating that foam cells had formed (Figure 1A). We also found that ART pretreatment effectively reduced ox-LDL-induced foam cell formation in THP-1-derived macrophages in a dose-dependent manner (Figure 1A).

In addition, lipid accumulation and intracellular total cholesterol analysis showed that ox-LDL loading could significantly increase lipid accumulation and intracellular cholesterol levels, while ART pretreatment effectively decreased ox-LDL-induced lipid accumulation (Figure 1B) and intracellular cholesterol accumulation (Figure 1C) in THP-1-derived macrophages in a dose-dependent manner.

\section{ART increased the gene and protein expression levels of ABCA1 and ABCG1 in THP-1-derived macrophages}

It is well known that VLDL-R, SRs, CD36, and class SR-A are critical for internalizing ox-LDL; ABCA1, ABCG1, and SR-BI mediate the efflux of cholesterol, and cholesterol esterification is mainly regulated by ACAT1 and nCEH $(3,4)$. Meanwhile, ABCA1, ABCG1, and SR$\mathrm{BI}$ are mainly responsible for the outflow of cholesterol in macrophages. As a physiological response to maintain cholesterol homeostasis, ox-LDL can up-regulate these reverse cholesterol transporters $(16,17)$. In experimental rodents or humans, the loss or impairment of ABCA1, ABCG1, or SR-BI can promote foam cell formation, atherosclerotic lesions, and an overwhelming inflammatory response (18-20).

As shown in Figure 2, after treating macrophages with ox-LDL, the mRNA expression levels of almost all genes involved in lipid internalization, esterification, and efflux were increased to varying degrees.

In addition, ART increased the mRNA and protein expression of ABCA1 and ABCG1 in macrophages in a dose-dependent manner compared to that of the ox-LDL alone group; however, ART did not change SR-BI expression in macrophages compared to the ox-LDL alone group.

In addition, no significant differences in the mRNA expression levels of $V L D L-R, S R-A, C D 36, A C A T 1$, or $n C E H$ were observed compared to those of the ox-LDL alone group.

\section{ART decreased TLR4 gene and protein expression in ox- LDL-induced THP-1-derived macrophages}

Atherosclerosis is a chronic inflammatory disease of the arteries, and it is well known that activation of the TLR signaling pathway plays an important role in $A B C A 1$ and $A B C G 1$ gene expression (5-7). The TLR4-dependent induction of IRF3 signaling was reported to effectively inhibit liver $\mathrm{X}$ receptor (LXR)-dependent expression of genes, such as $A B C A 1$ and $A B C G 1$, which are critical in cholesterol efflux from macrophages (21). Therefore, we measured the effect of ART on TLR4 expression. As shown in Figure 3, ART decreased TLR4 gene (Figure 3A) and protein (Figure $3 B$ ) expression in ox-LDL-induced THP-1derived macrophages in a dose-dependent manner. 
A

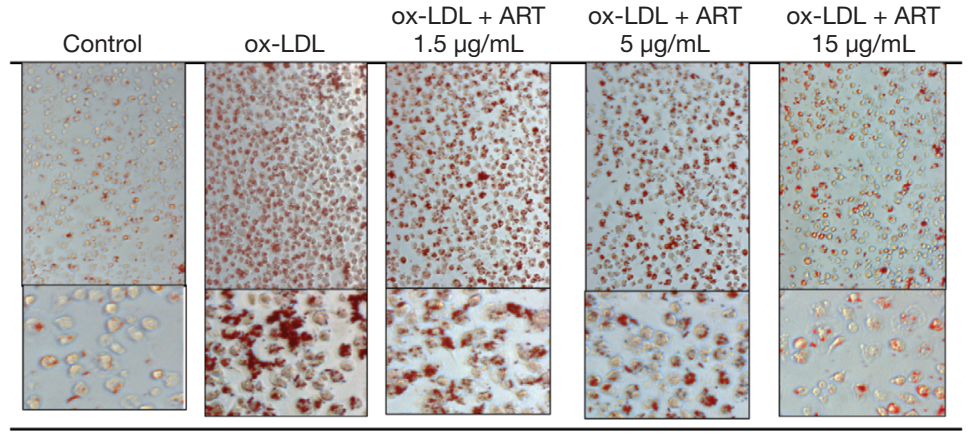

B

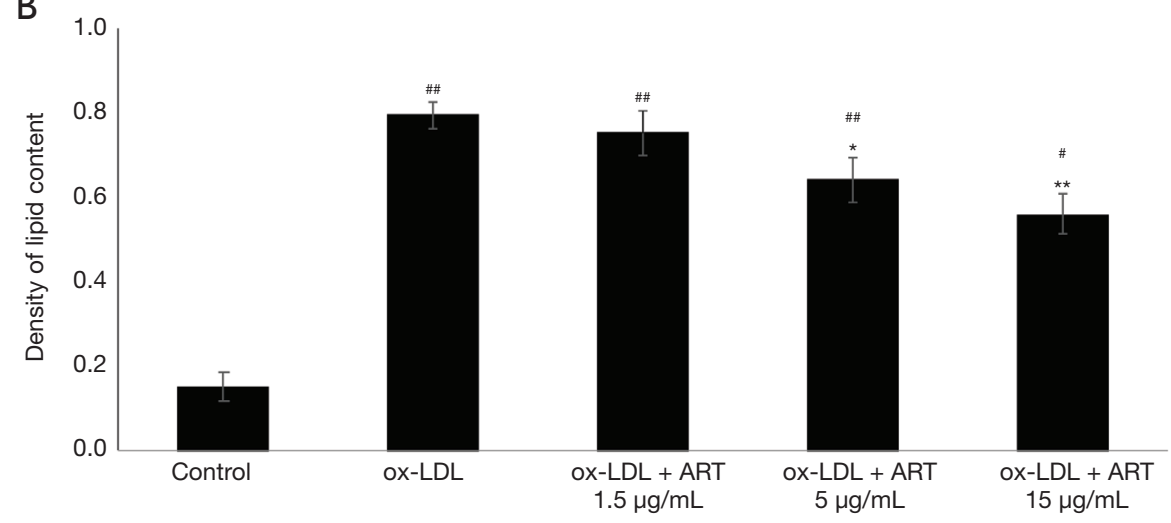

C

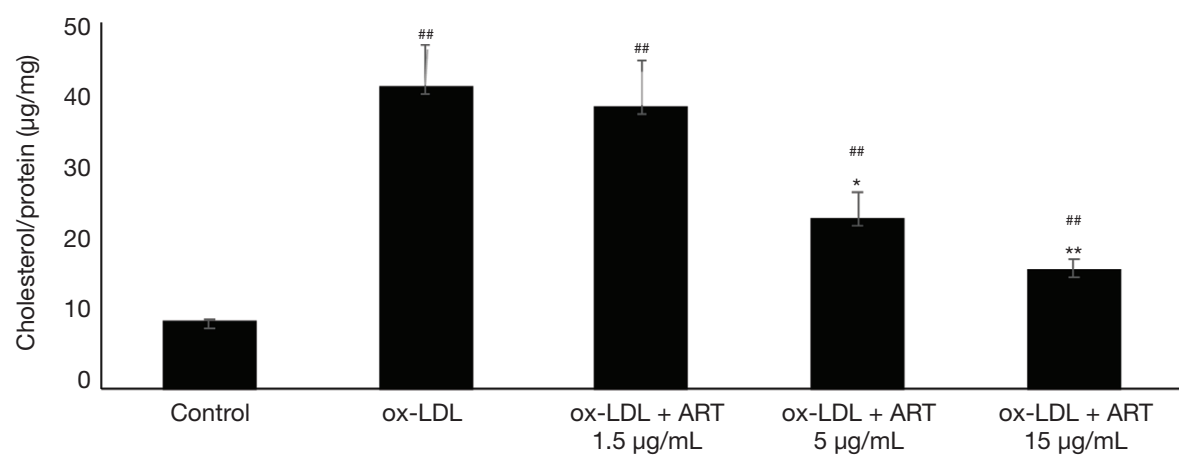

Figure 1 Effect of ART on macrophage-derived foam cell formation. (A) ART decreased the formation of foam cells from THP-1derived macrophages induced by ox-LDL (LM ×400). (B) ART decreased the ox-LDL-induced accumulation of lipids in THP-1-derived macrophages. (C) ART decreased the accumulation of cholesterol in THP-1-derived macrophages induced by ox-LDL. THP-1 derived macrophages were pretreated with ART $(0,1.5,5$, and $15 \mu \mathrm{g} / \mathrm{mL})$ for $12 \mathrm{~h}$, then incubated with ox-LDL $(100 \mu \mathrm{g} / \mathrm{mL})$ for $24 \mathrm{~h}$, and finally treated with oil red $\mathrm{O}$ staining (A), intracellular cholesterol quantification (B) and lipid quantification (C). The results are presented as the mean $\pm \mathrm{SD}$ of 3 independent experiments. ${ }^{\#} \mathrm{P}<0.05$ vs. the untreated control group, ${ }^{\# \#} \mathrm{P}<0.01$ vs. the untreated control group; ${ }^{*} \mathrm{P}<0.05$ vs. the ox-LDL alone group, ${ }^{* *} \mathrm{P}<0.01$ vs. the ox-LDL alone group. ART, artesunate; ox-LDL, oxidized low-density lipoprotein; SD, standard deviation. 


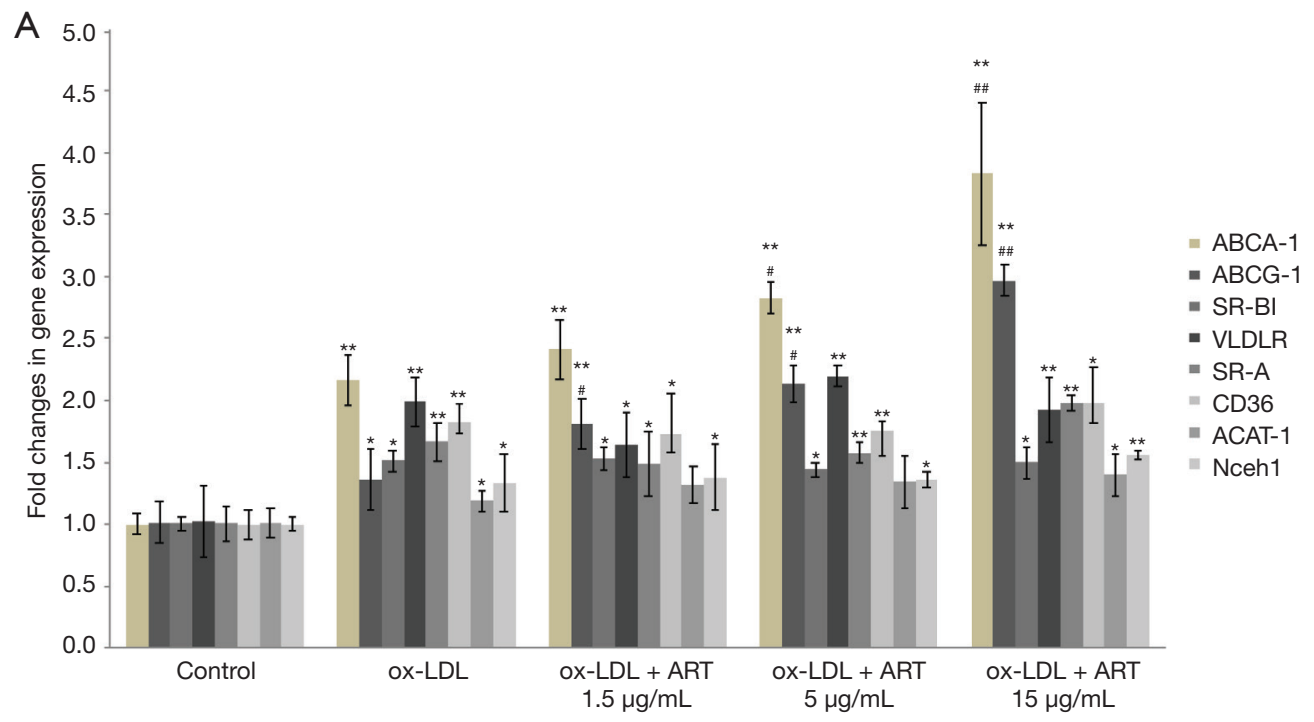

B

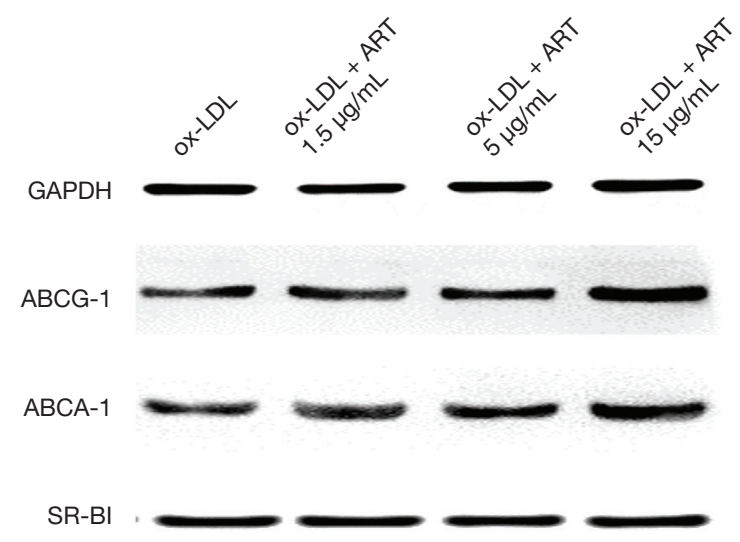

Figure 2 ART increased ABCA1 and ABCG1 expression in THP-1-derived macrophages induced by ox-LDL. (A) ABCA1 and ABCG1 gene expression. (B) ABCA1 and ABCG1 protein expression. Pretreated THP-1 derived macrophages with or without ART (0, 1.5, 5, and $15 \mu \mathrm{g} / \mathrm{mL})$ for $12 \mathrm{~h}$, then incubated with ox-LDL $(100 \mu \mathrm{g} / \mathrm{mL})$ for another $24 \mathrm{~h}$, and finally performed gene (A) and protein (B) expression analysis. The results are presented as the mean $\pm \mathrm{SD}$ of 3 independent experiments. ${ }^{\#} \mathrm{P}<0.05$ vs. the untreated control group, ${ }^{\# \#} \mathrm{P}<0.01$ vs. the untreated control group, ${ }^{*} \mathrm{P}<0.05$ vs. the ox-LDL alone group, ${ }^{* *} \mathrm{P}<0.05$ vs. the ox-LDL alone group. ART, artesunate; ox-LDL, oxidized low-density lipoprotein; SD, standard deviation.

\section{ART decreased IL- 6 and $T N F-\alpha$ release from ox- $L D L-$ induced THP-1-derived macrophages}

The activation of MAPK and NF- $\mathrm{B}$ pathways are mediated by TLR4, and it further activates a number of genes encoding pro-inflammatory cytokines and chemokines, such as IL- 6 and TNF- $\alpha$. Moreover, the activation of ABCA1 and ABCG1 also regulates the expression of inflammatory cytokines and chemokines in macrophages (18-20).
Therefore, we examined the effect of ART on IL-6 and TNF- $\alpha$ expression.

As shown in Figure 4, the levels of IL-6 and TNF- $\alpha$ in the supernatant in the control group were very low, and ox-LDL significantly increased the secretion of IL- 6 and TNF- $\alpha$ by THP-1-derived macrophages; furthermore, ART decreased IL- 6 and TNF- $\alpha$ secretion in a dosedependent manner. 


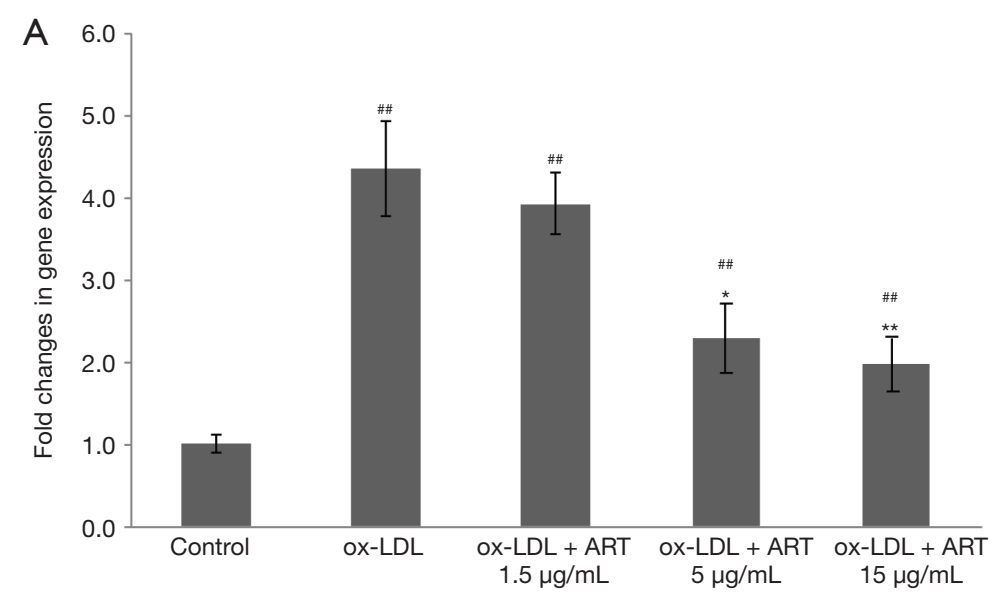

B

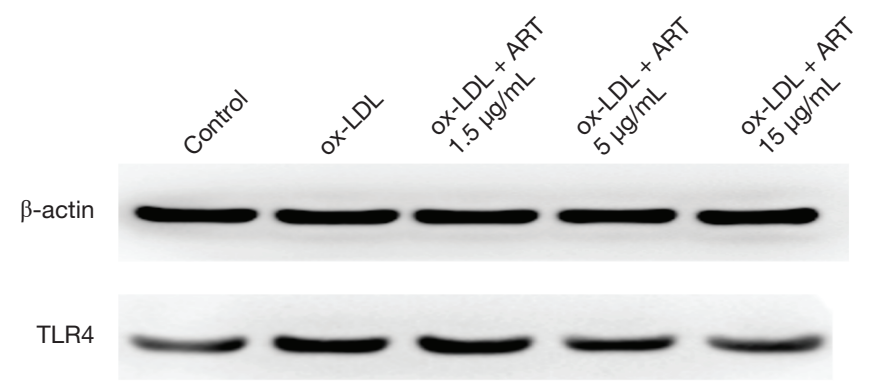

Figure 3 ART decreased TLR4 expression in THP-1-derived macrophages induced by ox-LDL. (A) TLR4 gene expression and (B) TLR4 protein expression. Pretreated THP-1 derived macrophages with or without ART $(0,1.5,5$, and $15 \mu \mathrm{g} / \mathrm{mL})$ for $12 \mathrm{~h}$, then incubated with ox-LDL (100 $\mu \mathrm{g} / \mathrm{mL})$ for another $24 \mathrm{~h}$, and finally performed gene (A) and protein (B) expression analysis. The results are presented as the mean $\pm \mathrm{SD}$ of 3 independent experiments. ${ }^{\#} \mathrm{P}<0.01$ vs. the untreated control group. ${ }^{*} \mathrm{P}<0.05$ vs. the ox-LDL alone group, ${ }^{* *} \mathrm{P}<0.01$ vs. the ox-LDL alone group. ART, artesunate; ox-LDL, oxidized low-density lipoprotein; SD, standard deviation.

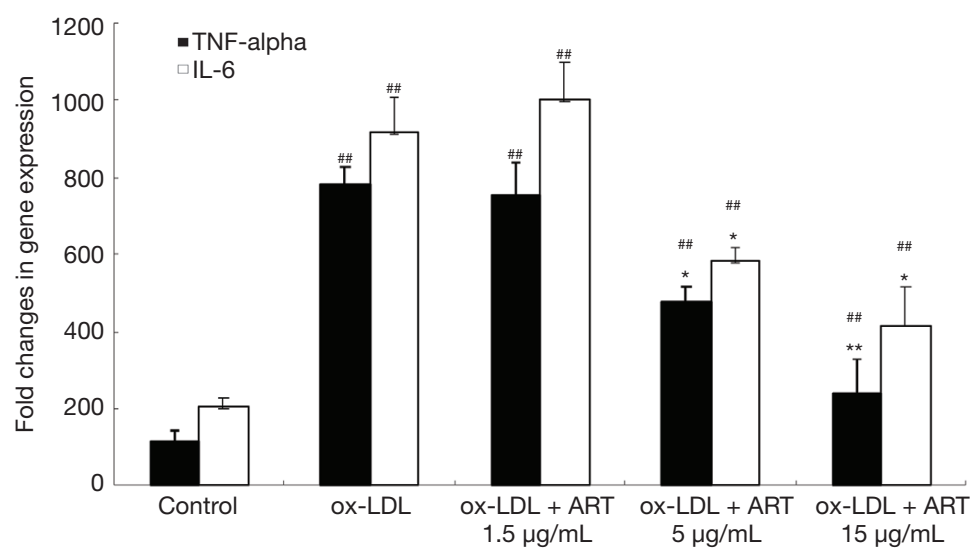

Figure 4 ART decreased IL-6 and TNF- $\alpha$ release from THP-1-derived macrophages induced by ox-LDL. THP-1-derived macrophages were pretreated with or without $\operatorname{ART}(0,1.5,5$ and $15 \mu \mathrm{g} / \mathrm{mL})$ for $12 \mathrm{~h}$ prior to incubation with ox-LDL $(100 \mu \mathrm{g} / \mathrm{mL})$ for an additional 24 $\mathrm{h}$, and then the supernatants were collected for cytokine analyses. The results are presented as the mean \pm SD of 3 independent experiments. ${ }^{\# \#} \mathrm{P}<0.01$ vs. the untreated control group. ${ }^{*} \mathrm{P}<0.05$ vs. the ox-LDL alone group, ${ }^{* *} \mathrm{P}<0.01$ vs. the ox-LDL alone group. ART, artesunate; oxLDL, oxidized low-density lipoprotein; SD, standard deviation; IL-6, interleukin 6; TNF- $\alpha$, tumor necrosis factor- $\alpha$. 


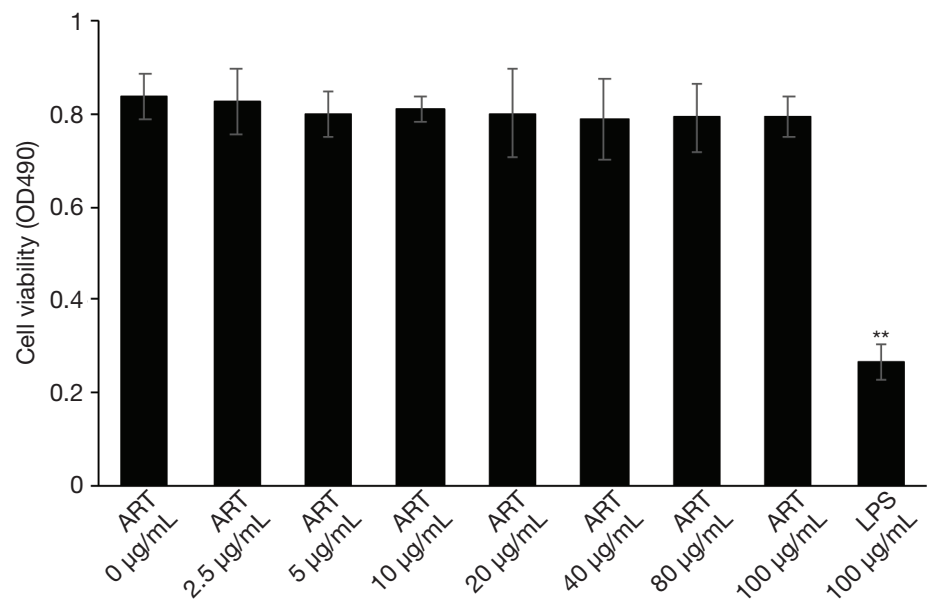

Figure 5 Artesunate has low cellular toxicity on THP-1-derived macrophages. THP-1-derived macrophages were incubated with ART $(0-100 \mu \mathrm{g} / \mathrm{mL})$ or $100 \mathrm{ng} / \mathrm{mL}$ LPS as a cytotoxic control for $24 \mathrm{~h}$. Cell viability was assessed by MTT assay. ${ }^{* *} \mathrm{P}<0.01$ vs. the ART $0 \mu \mathrm{g} / \mathrm{mL}$ group. ART, artesunate; LPS, lipopolysaccharide; MTT, 3-(4,5-dimethylthiazol-2-yl)-2,5-diphenyl tetrazolium bromide.

\section{ART has low cytotoxicity in vitro}

The cytotoxicity of ART on THP-1-derived macrophages were examined with an MTT assay. Treatment with ART $(0-100 \mu \mathrm{g} / \mathrm{mL})$ for $24 \mathrm{~h} \mathrm{did} \mathrm{not} \mathrm{induce} \mathrm{any} \mathrm{cytotoxicity} \mathrm{in}$ macrophages (Figure 5). These results indicated that the ART-induced effects were not related to cytotoxicity, since the concentration of ART used in our experiments was less than $100 \mu \mathrm{g} / \mathrm{mL}$.

\section{Discussion}

To our knowledge, this is the first report demonstrating that ART attenuates the formation of macrophage-derived foam cells. Cholesterol accumulation is decreased by ART through the enhancement of cholesterol efflux. The effects are related to decreases in ABCA1 and ABCG1, which might be related to the inhibition of TLR4.

Atherosclerosis is the main cause of many cardiovascular diseases and the second leading cause of death in elderly people $(1,2)$. The occurrence and development of atherosclerosis involves the interaction of activated monocytes with ox-LDL, which leads to the formation of foam cells $(4,22)$. Foam cells containing lipids form the initial basis of the lesion, and then the foam cells accumulate and produce fat streaks $(3,4)$. As the plaque develops, intermediate lesions are formed, which are characterized by smooth muscle cell proliferation and migration from the media to the intima. Late stages of plaque development include the formation of atherosclerotic plaques and fibroangiomas. In atheromatous plaques, foam cells aggregate and accumulate, eventually forming a necrotic core that is rich in lipids. Therefore, we considered that inhibiting foam cell formation could delay the progression of atherosclerosis.

Artemisinin is a commonly used antimalarial drug that was extracted from the Compositae Artemisia annua L. in 1971 by Chinese pharmacy workers. Due to the poor solubility, low bioavailability, and high reburning rate of artemisinin, scientists have developed various derivatives based on artemisinin; ART is one of these derivatives and is water-soluble $(8,9)$. Previous studies have reported that artemisinin and its derivatives could decrease cholesterol in plasma and triglycerides and reduce the areas of aortic root lesions $(23,24)$. However, our research revealed that ART could decrease the progression of atherosclerotic lesion formation in mice without effect on plasma lipid (14). In the present study, we revealed that ART could decrease the formation of macrophage-derived foam cells.

The imbalance in cholesterol influx, esterification, and efflux ultimately leads to the formation of foam cells. The SRs, SR-A, and CD36 are the main receptors responsible for the binding and uptake of ox-LDL; ACAT1 and nCEH play important roles in cholesterol esterification; and ABCA1, ABCG1, and SR-BI mediate cholesterol efflux $(3,4)$. When cholesterol efflux and esterification increase, or cholesterol outflow decreases, macrophages eventually become lipid-rich foam cells. Recent studies have shown 
that some traditional Chinese medicines and/or their extracts, such as curcumin, purple perilla, tanshinone IIA, and astragalin exhibit an anti-atherosclerotic effect, which is closely related to an increase in cholesterol efflux (25-27). Excess cholesterol in macrophage-derived foam cells is removed by high-density lipoprotein (HDL) and its major component apolipoprotein apoA-1 and is considered to be one of the key mechanisms of HDL-mediated atherosclerotic protection $(28,29)$.

The efflux of cholesterol in macrophages is mainly responsible for ABCA1, ABCG1, and SR-BI. The active transport of intracellular cholesterol and phospholipids to apoA-1 is mediated by ABCA1, and the removal of cholesterol from macrophages to HDL particles is mediated by ABCG1, but it does not mediate the removal of lipid apoA-1 (28). Absence or damage of ABCA1, ABCG1, or SR-BI function can promote the accumulation of foam cells, leading to atherosclerotic lesions and excessive inflammation (30-32). As previously demonstrated, curcumin can mediate the increase in cholesterol efflux in macrophages by heme oxygenase-1-mediated ABCA1 and SR-BI, while perilla extract and $\alpha$-asarone can also increase ox-LDL-induced cholesterol efflux in macrophages (25). In the present study, we found that ART increased the ox-LDL-induced expression of ABCA1 and ABCG1 in THP-1-derived macrophages without effect on the SR-BI. Therefore, we concluded that ART primarily attenuates the formation of foam cells through the inhibition of ABCA1/ ABCG1-dependent cholesterol efflux.

Next, we aimed to reveal the upstream mechanism by which ART induces the expression of ABCA1 and ABCG1. In our previous studies, we found that ART decreased the expression of TLR4 in macrophages (14). The TLRs are type 1 transmembrane receptors that play key roles in host recognition and defense against microbial infections. Moreover, many evidences suggest that the mechanism of bacterial-induced foam cell formation is closely related to PAMP-mediated TLR-dependent signaling, which plays an important role in atherogenesis (33). As a member of the TLR family, TLR4 is expressed in several types of cells, including neutrophils, macrophages, and dendritic cells, and it is also involved in the development of CAD (27,33-35). A recent report showed that TLR4 plays an important role in the accumulation of early intimal foam cells in the aorta of ApoE-/- TLR4-/- mice. Compared with ApoE-/- mice, the level of intimal lipids reduced by $75 \%$ (36).

Researchers have conducted further studies on how TLR-dependent signaling promotes cholesterol accumulation in macrophages (35). Yin et al. found that TLR4-mediated inflammation could dysregulate ACAT1 expression and promote foam cell formation in vascular smooth muscle cells (37). Researchers found that in cultured macrophages and aortic tissue in vivo, microbial ligands blocked the induction of LXR target genes (including ABCA1) by activating TLR4. Moreover, TLR4dependent induction of IRF3 signaling potently inhibits LXR-dependent expression of genes such as $A B C A 1$ and $A B C G 1$, which are involved in the efflux of cholesterol from macrophages $(38,39)$. Therefore, we hypothesized that inhibiting TLR4 could increase the expression of ABCA1 and ABCG1, ultimately increasing cholesterol efflux. Then, we concluded that ART could influence TLR4-mediated ABCA1-dependent and ABCG1-dependent cholesterol efflux, regulating the formation of foam cells.

The TLRs play critical roles in regulating immunity. These receptors recruit adaptor molecules to mediate the activation of MAPKs and NF- $\kappa$ B pathways, which in turn activates many genes encoding pro-inflammatory cytokines and chemokines, such as TNF- $\alpha$, IL-6, and IL-1 (40). Furthermore, recent studies have shown that the sterol efflux activities of ABCA1 and ABCG1 can regulate macrophage expression of inflammatory cytokines and chemokines, as well as the proliferative responses of lymphocytes $(39,41)$. In the present study, we found that ART further decreased IL- 6 and TNF- $\alpha$ release from oxLDL-induced THP-1-derived macrophages, which might result in a decrease in TLR4 expression and an increase in ABCA1 and ABCG1 expression.

\section{Conclusions}

In conclusion, ART attenuated the formation of ox-LDLinduced foam cells from THP-1-derived macrophages by increasing $\mathrm{ABCA} 1$ and $\mathrm{ABCG} 1$ expression via inhibition of TLR4 expression and reduction of TNF- $\alpha$ and IL- 6 secretion from macrophages induced by ox-LDL, which ultimately decreased the accumulation of cholesterol. ART is worthy of further research as a drug candidate for the treatment of atherosclerosis.

\section{Acknowledgments}

Funding: This study was supported by the National Science Foundation of China (81703568) and the Kuanren Talents Program of the second affiliated hospital of Chongqing Medical University (13-004-027). 


\section{Footnote}

Reporting Checklist: The authors have completed the MDAR reporting checklist. Available at https://dx.doi. org/10.21037/atm-21-3551

Data Sharing Statement: Available at https://dx.doi. org/10.21037/atm-21-3551

Conflicts of Interest: All authors have completed the ICMJE uniform disclosure form (available at https://dx.doi. org/10.21037/atm-21-3551). The authors have no conflicts of interest to declare.

Ethical Statement: The authors are accountable for all aspects of the work in ensuring that questions related to the accuracy or integrity of any part of the work are appropriately investigated and resolved.

Open Access Statement: This is an Open Access article distributed in accordance with the Creative Commons Attribution-NonCommercial-NoDerivs 4.0 International License (CC BY-NC-ND 4.0), which permits the noncommercial replication and distribution of the article with the strict proviso that no changes or edits are made and the original work is properly cited (including links to both the formal publication through the relevant DOI and the license). See: https://creativecommons.org/licenses/by-ncnd/4.0/.

\section{References}

1. Liu M, Zheng Y, Li G. Safety of Recanalization Therapy in Patients with Acute Ischemic Stroke Under Anticoagulation: A Systematic Review and Meta-Analysis. J Stroke Cerebrovasc Dis 2018;27:2296-305.

2. Ellulu MS, Patimah I, Khaza'ai H, et al. Atherosclerotic cardiovascular disease: a review of initiators and protective factors. Inflammopharmacology 2016;24:1-10.

3. Duraisamy P, Ravi S, Krishnan M, et al. Dynamic Role of Macrophage Sub Types for Development of Atherosclerosis and Potential Use of Herbal Immunomodulators as Imminent Therapeutic Strategy. Cardiovasc Hematol Agents Med Chem 2020. [Epub ahead of print]. doi: 10.21 74/1871525718666201217163207.

4. Sukhorukov VN, Khotina VA, Chegodaev YS, et al. Lipid Metabolism in Macrophages: Focus on Atherosclerosis.
Biomedicines 2020;8:262.

5. Ministrini S, Carbone F, Montecucco F. Updating concepts on atherosclerotic inflammation: From pathophysiology to treatment. Eur J Clin Invest 2021;51:e13467.

6. Chen X, Zhao D, Ning Y, et al. Toll-like receptors 2 expression in mediastinal lymph node of patients with sarcoidosis. Ann Transl Med 2020;8:1182.

7. Roshan MH, Tambo A, Pace NP. The Role of TLR2, TLR4, and TLR9 in the Pathogenesis of Atherosclerosis. Int J Inflam 2016;2016:1532832.

8. Tu Y. Artemisinin-A Gift from Traditional Chinese Medicine to the World (Nobel Lecture). Angew Chem Int Ed Engl 2016;55:10210-26.

9. Duarte D, Vale N. New Trends for Antimalarial Drugs: Synergism between Antineoplastics and Antimalarials on Breast Cancer Cells. Biomolecules 2020;10:E1623.

10. Mancuso RI, Foglio MA, Olalla Saad ST. Artemisinin-type drugs for the treatment of hematological malignancies. Cancer Chemother Pharmacol 2021;87:1-22.

11. Raffetin A, Bruneel F, Roussel C, et al. Use of artesunate in non-malarial indications. Med Mal Infect 2018;48:238-49.

12. Liu $\mathrm{X}, \mathrm{Wu} \mathrm{J}$, Wang $\mathrm{N}$, et al. Artesunate reverses LPS tolerance by promoting ULK1-mediated autophagy through interference with the CaMKII-IP3R-CaMKK $\beta$ pathway. Int Immunopharmacol 2020;87:106863.

13. Sordi R, Nandra KK, Chiazza F, et al. Artesunate Protects Against the Organ Injury and Dysfunction Induced by Severe Hemorrhage and Resuscitation. Ann Surg 2017;265:408-17.

14. Jiang W, Cen Y, Song Y, et al. Artesunate attenuated progression of atherosclerosis lesion formation alone or combined with rosuvastatin through inhibition of proinflammatory cytokines and pro-inflammatory chemokines. Phytomedicine 2016;23:1259-66.

15. Jiang $\mathrm{W}, \mathrm{Li} \mathrm{B}$, Zheng $\mathrm{X}$, et al. Artesunate in combination with oxacillin protect sepsis model mice challenged with lethal live methicillin-resistant Staphylococcus aureus (MRSA) via its inhibition on proinflammatory cytokines release and enhancement on antibacterial activity of oxacillin. Int Immunopharmacol 2011;11:1065-73.

16. Choromanska B, Mysliwiec P, Hady HR, et al. The implication of adipocyte ATP-binding cassette A1 and G1 transporters in metabolic complications of obesity. J Physiol Pharmacol 2019. doi: 10.26402/jpp.2019.1.14.

17. Chistiakov DA, Melnichenko AA, Myasoedova VA, et al. 
Mechanisms of foam cell formation in atherosclerosis. J Mol Med (Berl) 2017;95:1153-65.

18. Peng Y, Xu J, Zeng Y, et al. Polydatin attenuates atherosclerosis in apolipoprotein E-deficient mice: Role of reverse cholesterol transport. Phytomedicine 2019;62:152935.

19. Zou TB, Zhu SS, Luo F, et al. Effects of Astaxanthin on Reverse Cholesterol Transport and Atherosclerosis in Mice. Biomed Res Int 2017;2017:4625932.

20. Zeng Y, Peng Y, Tang K, et al. Dihydromyricetin ameliorates foam cell formation via LXR $\alpha$-ABCA1/ ABCG1-dependent cholesterol efflux in macrophages. Biomed Pharmacother 2018;101:543-52.

21. Ma L, Dong F, Zaid M, et al. ABCA1 protein enhances Toll-like receptor 4 (TLR4)-stimulated interleukin-10 (IL10) secretion through protein kinase A (PKA) activation. J Biol Chem 2012;287:40502-12.

22. Corti A, Gaucher C, Pompella A. Editorial: Targeting Monocytes/Macrophages to Treat Atherosclerotic Inflammation. Front Pharmacol 2020;11:86.

23. He LH, Gao JH, Yu XH, et al. Artesunate inhibits atherosclerosis by upregulating vascular smooth muscle cells-derived LPL expression via the KLF2/NRF2/ TCF7L2 pathway. Eur J Pharmacol 2020;884:173408.

24. Cao Q, Du H, Fu X, et al. Artemisinin Attenuated Atherosclerosis in High-Fat Diet-Fed ApoE-/- Mice by Promoting Macrophage Autophagy Through the AMPK/mTOR/ULK1 Pathway. J Cardiovasc Pharmacol 2020;75:321-32.

25. Zhong Y, Liu C, Feng J, et al. Curcumin affects oxLDL-induced IL-6, TNF- $\alpha$, MCP-1 secretion and cholesterol efflux in THP-1 cells by suppressing the TLR4/NF- $\mathrm{B} / \mathrm{miR} 33$ a signaling pathway. Exp Ther Med 2020;20:1856-70.

26. Tan YL, Ou HX, Zhang M, et al. Tanshinone IIA Promotes Macrophage Cholesterol Efflux and Attenuates Atherosclerosis of apoE-/- Mice by Omentin-1/ABCA1 Pathway. Curr Pharm Biotechnol 2019;20:422-32.

27. Zhao ZW, Zhang M, Wang G, et al. Astragalin Retards Atherosclerosis by Promoting Cholesterol Efflux and Inhibiting the Inflammatory Response via Upregulating ABCA1 and ABCG1 Expression in Macrophages. J Cardiovasc Pharmacol 2021;77:217-27.

28. Wang N, Westerterp M. ABC Transporters, Cholesterol Efflux, and Implications for Cardiovascular Diseases. Adv Exp Med Biol 2020;1276:67-83.
29. Garg PK, Jorgensen NW, McClelland RL, et al. Cholesterol mass efflux capacity and risk of peripheral artery disease: The Multi-Ethnic Study of Atherosclerosis. Atherosclerosis 2020;297:81-6.

30. Linton MF, Tao H, Linton EF, et al. SR-BI: A Multifunctional Receptor in Cholesterol Homeostasis and Atherosclerosis. Trends Endocrinol Metab 2017;28:461-72.

31. Ren Q, Xie X, Tang Y, et al. Methyl tertiary-butyl ether inhibits THP-1 macrophage cholesterol efflux in vitro and accelerates atherosclerosis in ApoE-deficient mice in vivo. J Environ Sci (China) 2021;101:236-47.

32. Ma J, Zhao D, Wang X, et al. LongShengZhi Capsule Reduces Established Atherosclerotic Lesions in apoEDeficient Mice by Ameliorating Hepatic Lipid Metabolism and Inhibiting Inflammation. J Cardiovasc Pharmacol 2019;73:105-17.

33. Li B, Xia Y, Hu B. Infection and atherosclerosis: TLRdependent pathways. Cell Mol Life Sci 2020;77:2751-69.

34. Li Y, Wang Y, Chen Y, et al. Corilagin Ameliorates Atherosclerosis in Peripheral Artery Disease via the TollLike Receptor-4 Signaling Pathway in vitro and in vivo. Front Immunol 2020;11:1611.

35. Zhang Y, Zhao Q. AEG-1 deletion promotes cartilage repair and modulates bone remodeling-related cytokines via TLR4/MyD88/NF- $\mathrm{B}$ inhibition in ovariectomized rats with osteoporosis. Ann Transl Med 2020;8:1298.

36. Higashimori M, Tatro JB, Moore KJ, et al. Role of tolllike receptor 4 in intimal foam cell accumulation in apolipoprotein E-deficient mice. Arterioscler Thromb Vasc Biol 2011;31:50-7.

37. Yin YW, Liao SQ, Zhang MJ, et al. TLR4-mediated inflammation promotes foam cell formation of vascular smooth muscle cell by upregulating ACAT1 expression. Cell Death Dis 2015;6:1659.

38. Zhao L, Lei W, Deng C, et al. The roles of liver X receptor $\alpha$ in inflammation and inflammation-associated diseases. J Cell Physiol 2021;236:4807-28.

39. Mogilenko DA, Kudriavtsev IV, Trulioff AS, et al. Modified low density lipoprotein stimulates complement $\mathrm{C} 3$ expression and secretion via liver $\mathrm{X}$ receptor and Tolllike receptor 4 activation in human macrophages. J Biol Chem 2012;287:5954-68.

40. Ramji DP, Davies TS. Cytokines in atherosclerosis: Key players in all stages of disease and promising therapeutic targets. Cytokine Growth Factor Rev 2015;26:673-85. 
41. Wang H, Li Y, Zhang X, et al. DPP-4 Inhibitor Linagliptin Ameliorates Oxidized LDL-Induced THP1 Macrophage Foam Cell Formation and Inflammation.
Drug Des Devel Ther 2020;14:3929-40.

(English Language Editor: J. Jones)

Cite this article as: Qian Y, Xia L, Wei L, Jiang W. Artesunate attenuates foam cell formation by enhancing cholesterol efflux. Ann Transl Med 2021;9(17):1379. doi: 10.21037/atm-21-3551 